\title{
Job Shop Scheduling Based on Digital Twin Technology: A Survey and an Intelligent Platform
}

\author{
Haifei Yu $\mathbb{D}^{1},{ }^{1}$ Songjian Han, ${ }^{1}$ Dongsheng Yang, ${ }^{2}$ Zhiyong Wang $\mathbb{D}^{3},{ }^{3}$ and Wei Feng $\mathbb{D}^{4}$ \\ ${ }^{1}$ School of Business Administration, Northeastern University, Shenyang, China \\ ${ }^{2}$ College of Information Science and Engineering, Northeastern University, Shenyang, China \\ ${ }^{3}$ Shenzhen Institutes of Advanced Technology, Chinese Academy of Sciences, Beijing, China \\ ${ }^{4}$ Shenzhen Key Laboratory of Smart Sensing and Intelligent Systems, Shenzhen, China \\ Correspondence should be addressed to Haifei Yu; hfyu@mail.neu.edu.cn
}

Received 6 August 2020; Revised 16 September 2020; Accepted 26 March 2021; Published 15 April 2021

Academic Editor: Atila Bueno

Copyright (C 2021 Haifei Yu et al. This is an open access article distributed under the Creative Commons Attribution License, which permits unrestricted use, distribution, and reproduction in any medium, provided the original work is properly cited.

\begin{abstract}
The concept of digital twinning has become a hot topic in the manufacturing industry in recent years. The emerging digital twin technology is an intelligent technology that makes full use of multimodels, big data, and interdisciplinary knowledge, which provides some new approaches for the field of the intelligent manufacturing industry. The job shop scheduling problem has been an important research field in the discrete manufacturing industry. Digital twin technology is adopted to solve the problem of job shop scheduling, which provides the possibility for the intelligent development of workshops. Based on digital twin technology and combined with the actual problem of production line scheduling, we propose a new intelligent scheduling platform to solve the shop scheduling problems above. Meanwhile, based on the prediction and diagnosis of multisource dynamic interference in the workshop production process by big data analysis technology, the corresponding interference strategy is formulated in advance by the scheduling cloud platform. The model simulation experiment of intelligent dispatching cloud platform was carried out, and some enterprises in intelligent manufacturing workshop were taken as examples to verify the superiority of the dispatching cloud platform. Finally, we look forward to the future research direction of intelligent manufacturing based on digital twin technology.
\end{abstract}

\section{Introduction}

The competition among manufacturing enterprises is more and more intense, and manufacturing enterprises pay more and more attention to improve their competitiveness through an efficient workshop production scheduling system. Although a lot of theoretical achievements have been made in the study of classical job shop scheduling problems, the established models are far from the actual production situation, so it is difficult to guide the production practice. In recent years, the research on the real-time scheduling problem of job shops has become a hot spot in the field of manufacturing system research. Digital twinning is considered an effective solution [1]. The implementation of the digital twin is a process in which virtual space and physical space of the product life cycle promote each other. The digital twin can directly compare the theoretical value of big data analysis with the actual value of product life cycle activities. As a result, it iteratively optimizes various activities throughout the product lifecycle. In digital twin's virtual space, various activities throughout the product life cycle can be simulated, monitored, optimized, and validated. The workshop scheduling cloud platform based on digital twin technology effectively realizes the seamless coordination of the entire product life cycle, becomes a bridge connecting the physical world and the network world, provides a new way for manufacturing enterprises to realize intelligent production and refined management, and can effectively avoid information islands and data duplication [2]. Digitalization of the entire manufacturing plant is possible because the physical process is closely linked to the network process. In the design of the product model, if the design 
scheme cannot pass the simulation test, the design scheme must be redesigned in real time. When a product is redesigned, big data was used to find problems and the design was improved. The required tools and algorithms were used as services during product design. In the manufacturing stage, the available resources are quickly searched through the matching and scheduling of supply and demand of manufacturing resource service [3] based on super network. On this basis, all required resources are integrated for analysis and planning through big data analysis. The production plan was simulated, evaluated, and improved. After getting the best production plan, it is delivered to the real world for actual production. At the same time, real-time data is collected from the physical world, virtual models are built, production processes are monitored, and plans are compared. If there is a difference, big data analysis was used to find out the reason and formulate a solution, such as adjusting equipment or improvement plan. In iterative interactions, production can be fully realized according to the optimal scheme. In addition, once the design changes, the manufacturing process can be easily updated, including updating material lists, processes, and allocating new resources. Therefore, the integration of digital twinning, big data, and services makes it possible to optimize production plans and adjust manufacturing processes in real time [4]. In the daily operation and Maintenance, Repair, and Operations (MRO) of the product, the virtual model of the physical product is synchronized with the real state of the product through sensors. We master the running state of the product and the running state of each component in real time. In addition to sensor data, digital twin integrates historical data (for example, maintenance records and energy consumption records). Through big data analysis of the above data, the product digital twin can continuously predict the health status, remaining life, and failure probability of the product.

With the help of big data analysis, product digital twins can also reveal unknown problems by comparing actual product responses and predicting specific scenarios of product responses. Once hidden trouble or failure is found; the maintenance program is simulated and optimized in the virtual world for practical maintenance. Similarly, the resources and capabilities, tools, and algorithms required for day-to-day operations and MRO phases are used as services [5]. Big data analytics is responsible for analyzing all the data needed for intelligent manufacturing, and digital twins make up for the lack of big data's ability to simulate and synchronously visualize physical processes. Therefore, the integration of digital twinning, big data, and services is of great significance for intelligent manufacturing.

The rest of the paper is organized as follows. The evolution process and application scenario of digital twin technology are presented in Section 2, followed by the fusion of digital twinning technology and the shop scheduling problem proposed in Section 3. The framework model of intelligent scheduling platform in intelligent manufacturing workshop based on digital twinning is illustrated and a case study is presented in Section 4. Then, related outlook contents are presented in Section 5, which also concludes the paper.

\section{Literature Review}

2.1. Digital Twin Technology Concept and Application in CPS. The concept of the digital twin was first proposed in the Product Lifecycle Management (PLM) course taught by American scholar Grieves in 2003, and the digital twin was defined as a combination of a set of virtual models and can depict potential or real physical manufacturing products completely from micro to macro. It already includes all the elements of digital twinning: real space, virtual space, and information interaction between real space and virtual space data streams [6]. The concept is also in the process of continuous improvement and development, has been widely used in the industrial field, and has verified its substantial potential [7]. Digital twinning is an intelligent technology, which uses the digital virtual model to reflect the physical model working behavior in the real environment and expands the new capability of physical models by means of data interaction, information fusion, and iterative optimization, and so on.

The terms "intelligent manufacturing" and "industry 4.0" have been used interchangeably in academic literature and in actual industrial production and are now almost synonymous. Its main goal is to leverage the latest advances in information and communication technologies to achieve autonomous, self-optimizing, and self-diagnostic capabilities to alleviate problems in complex manufacturing scenarios. Digital twins are typically used in the context of network physical systems to reflect the life of their real objects through the best physical models and sensor data. As a result, the digital twin can simulate the real world in the network world, which would otherwise take a lot of resources and time. Figure 1 shows our proposed digital twin architecture for the Cyber-Physical System (CPS). CPS is a mechanism for intelligent manufacturing and industry 4.0 design principles. It is made up of five main parts:

(1) The factory: The physical workshop must be connected to the Internet for relevant data collection. The equipment information, material handling information, and warehouse information in the production process can be transmitted to the twin workshop. The system needs to interact with the workshop real-time data and feedback to the cloud.

(2) The digital twin technology and its runtime environment: Using digital twinning technology, the optimal time point for maintenance can be predicted through the continuous collection and intelligent analysis of operation data.

(3) The factory interface extracts the sensor/actuator data from physical space: in the production environment of network space, process modeling and simulation are carried out for the workflow and efficiency in each production unit.

(4) The application program interface: It provides the application programming interface (API) for applications wishing to take advantage of the digital twin technology. 


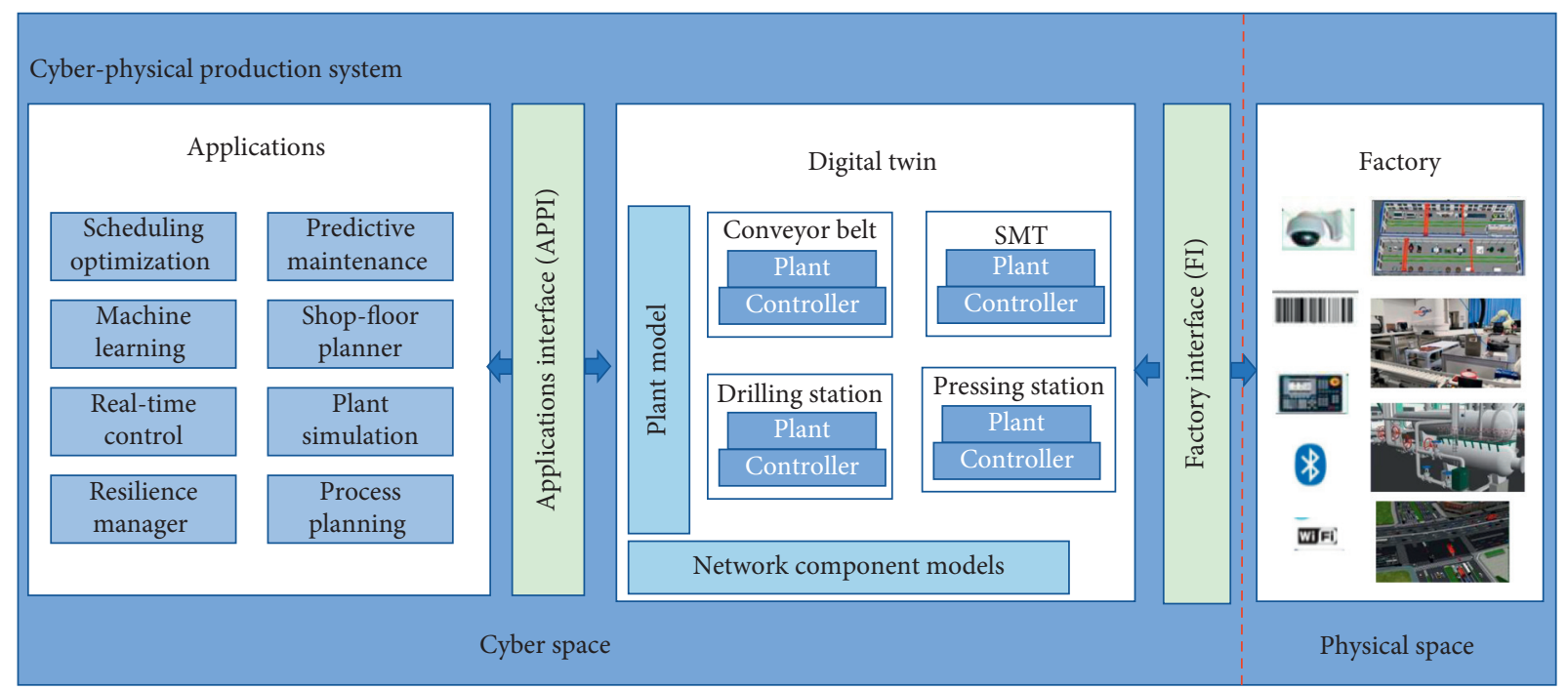

FIgURE 1: Digital twin in CPS.

(5) The application itself: Command and coordination hub center, coordinate resource allocation, preventive maintenance management of equipment, machine learning, self-driven simulation, process planning and scheduling, and timely feedback model establishment, production, and processing process control.

\subsection{Featured Application Scenarios of Digital Twin}

2.2.1. Digital Twin Applied to the Digital Product Development. Most of the product research and development costs are locked in the concept design phase, and the early rapid iteration becomes critical with the aid of digital twin. It helps the users with less cost and faster speed of digital twin applying innovative technology to the market, and the users can use structure thermal electromagnetic fluid and control simulation software such as a separate item field and coupled physics field research, so as to realize the design optimization of product verification and validation to meet the relevant requirements [8].

\subsubsection{Digital Twin Applied to Process Planning. With the} application of digital twins, systematic planning can be carried out for the products that need to be manufactured, as well as the manufacturing methods, resources, and locations, etc., and all aspects can be linked together to realize the collaboration between designers and manufacturers [9]. Once a product design change occurs, the manufacturing process can be easily updated in the digital twin model. In addition to process planning, production layout is also an important problem for intelligent manufacturing systems. With the help of the digital twin model, it is possible to design a production layout that contains all the detailed information, including machinery, automation, equipment, tools, resources, and even operators, and seamlessly relate it to the product design.
2.2.3. Digital Twin Applied to Lean Manufacturing. With the application of digital twin, different manufacturing strategies can be simulated and evaluated. Combining big data technology and statistical analysis, the processes with free time can be quickly found [10]. After adjusting the strategy, the performance of the whole manufacturing system is simulated to further optimize the utilization of all resources.

2.2.4. Digital Twin Applied to Energy and Mining Solutions. Digital twin technology is a real-time virtual version of the actual running device and can be used to provide performance and maintenance information for the product [4]. For example, in the design, construction, operation, and supervision of a large railway project, there are many problems: the railway line leads to problems such as design slopes to ensure safe construction. Choosing the right place to drill the hole can reduce the occurrence of landslides effectively. Optimizing process logic can effectively reduce cost and guarantee quality. Completion strategies were developed to minimize energy consumption. Reasonable allocation of machine equipment load effectively prolongs the service life of equipment assets. Digital twin technology can effectively solve the above problems: through a variety of sensors on the equipment, such as temperature, vibration, impact, load data, and real-time input digital twin model, so that the digital environment model of the duplex and the change of the actual equipment working environment keep consistent. Digital twin provides predictability in the equipment state and can replace worn parts during scheduled shutdowns to avoid unplanned shutdowns.

\subsubsection{Digital Twin Applied to the Construction of Smart City.} In recent years, some countries have applied digital twin to the construction of smart cities. For example, in 2016, City Scope, there was a collaboration between Singapore and the Massachusetts Institute of Technology, tailor-made city operation simulation system for Singapore's urban planning. 
With the support of 8 million Euros from the European Union, Spanish smart Santander deployed various sensors in cities to sense the urban environment, traffic, water conservancy, and other operating conditions and gathered data into the urban dashboard of the smart city platform, initially forming the prototype of digital twin cities and becoming one of the templates that can be promoted in Europe [11]. At present, digital twins are mainly applied in urban planning and management and will be extended to urban services in the future. Through the construction of digital twins system in service scenarios, service objects, and service contents, the service mode will be accelerated to transform to the combination of virtual and real blending of scenes, personalization, and activeness.

2.3. Workshop Scheduling. In the single-objective dynamic scheduling stage, Imen et al. [12] aimed at minimizing the maximum completion time, improved the existing problems in coding, crossover, and variation in the traditional genetic algorithm, and proposed an improved genetic algorithm to solve the dynamic shop scheduling problem. Wang et al. [13] designed the genetic algorithm in two stages with the maximum completion time as the optimization target. Based on the improved genetic algorithm, the scheduling problem of uncertain processing time in the flexible job shop is solved. In the phase of multiobjective dynamic adjustment, Addona et al. [14] used the traditional algorithm to solve the multiobjective mathematical model based on cost, quality, and processing time. Liu et al. [15] established a mathematical model with minimum processing time and minimum workpiece delay as the optimization objectives and proposed to solve the problem by using an adaptive genetic algorithm to optimize the multiobjective dynamic flexible workshop scheduling problem [16].

In recent days, some experts and scholars began to study the reliability prediction of workshop equipment and the monitoring and capture of workshop disturbance. Zhang et al. [17] proposed an intelligent bearing reliability diagnosis method with automatic learning function. Based on the subsets of the depth autoencoder (SBTDA) model, the automatic extraction of discriminant features of different reliability modes is realized, and the intelligent predictive diagnosis of bearing reliability is realized. Ding et al. [18] proposed a social manufacturing system (RFID-SMS) based on radio frequency identification to realize real-time monitoring of interenterprise/interfactory/workshop production scheduling tasks. RFID devices are systematically deployed in each enterprise's workshop equipment to collect real-time production and transportation data [19].

2.4. Summary of Literature. In 1990, Bucker and Schlie first proposed that, in the job shop scheduling problem, the machining process of the job shop could be processed on different machines and designed a polynomial algorithm to solve this problem, which was the first time that Flexible Job Scheduling problem (FJSP) was proposed [20]. Subsequently, Holsapple designed the genetic coding of machine numbers and sequences of each process to solve this problem and used the FBS (Filtered Beam Search) search method to schedule and sort them [21]. Brandimarte uses a hierarchical approach using dispatch rules and tabu searches. The method to solve FJSP has obtained a good result [22]. Hurink applied the tabu search method and designed an integrated method to solve FJSP [23]. Kacem et al. designed a heuristic algorithm to initialize the multiobjective FJSP model and then used a genetic algorithm to optimize the initial solution, with good solution results [24]. DauzerePeres and Paulli adopt an integrated approach to solve FJSP by defining a neighborhood structure and using a tabu search algorithm. Liu Mingzhou et al. designed the hybrid PSO (particle swarm optimization) algorithm, combined with the coding rules of simulated annealing and genetic algorithm, and studied the multitarget FJSP [25]. According to the characteristics of FJSP, Zhang Chaoyong et al. improved the algorithm flow of the genetic algorithm and designed a two-pole genetic algorithm for the crossover strategy, which improved the search efficiency of the algorithm. Zhang Guohui et al. [15] designed a new generation method of the initial population of genetic algorithm for the FJSP problem, so that a high-quality population could be obtained in the initial phase of the algorithm under the premise of considering the balance of machine processing, thus improving the computational speed of the algorithm.

In the existing problems and research trends, the concept of cloud manufacturing has been widely accepted, but at present, the research on cloud manufacturing technology is still mainly focused on information technology rather than manufacturing technology, because it is not easy for managers to accept the transfer of manufacturing management system to the cloud in the actual production management. On the one hand, communication through the network platform is discontinuous, contrary to the continuous production situation in the production process. Management decision-makers, on the other hand, worry about whether production data is safe in the cloud. From the perspective of manufacturing resources themselves, compared with the general network information resources, manufacturing resources need more data to realize the process of virtualization and servitization, and then they are stored in the cloud platform. Manufacturing resources are highly specialized and require special expertise in the field of manufacturing, which is challenging for cloud manufacturing research based on technologies such as computers and the Internet. As the carrier of the cloud manufacturing process and the object of resource virtualization, cloud manufacturing service, therefore, needs further research and application verification. Based on this and combined with the above research review, the following problems can be concluded in the current research field of cloud manufacturing services:

(1) Research stage of digital twin technology: The existing research mainly focuses on the generation stage and platform use stage, with a few involving the evaluation stage. There is a lack of research on 
relevant models and technologies in the use and monitoring stage, elimination, and upgrade stage.

(2) Digital twin research depth of the manufacturing technology and intelligence in the production of whole life cycle and platform using phase: it is mainly the study of the resources, service virtualization, search and match, service composition and optimization, the study of these questions most at the concept and frame research stage, and less study on how to apply and implement.

(3) Digital twin research technologies and methods in cloud manufacturing services still depend on the number and scale of services, and the algorithm effect is not significant when the number of services does not reach a certain amount. The technologies and methods related to cloud manufacturing services provide the implementation process from scratch, and there are few literature studies on how to use the information of completed tasks and stored memory knowledge to simplify the implementation process.

At present, research studies on cloud manufacturing services mainly regard it as an important content and an object of cloud manufacturing, which has not yet played its role of actively creating value, and thus cannot strongly support in-depth researches on cloud manufacturing and intelligent manufacturing. Comprehensively considering user needs and applying more perfect methods or technologies will improve the content and expand the connotation of cloud manufacturing service research, thus improving the intelligent level of the manufacturing process and providing intelligent decision-making for the production process.

\section{Intelligent Scheduling Platform of Intelligent Manufacturing Workshop Based on Digital Twin}

\subsection{Intelligent Manufacturing Workshop Scheduling Intelli-} gent Platform Framework Model. Based on digital twin technology background, this paper proposes scheduling cloud platform concept in the intelligent manufacturing system, The existing production scheduling model and optimization algorithm model are transferred together with the obtained physical workshop production data to the realtime scheduling cloud platform of the intelligent manufacturing workshop. The integrated management scheduling model algorithm of intelligent Internet of Things and cloud computing technologies is applied to process the production data. Based on the need of actual production, scheduling a cloud platform needs digital twins with the following technical prerequisites:

(1) Virtual workshop modeling, simulation, and operation management technology based on digital twin technology, including the establishment of multidimensional model of "model-cons traction action" digital twin workshop, production factor management digital twin workshop, iteration and optimization of production process planning, and real-time interaction between the physical workshop and virtual workshop.

(2) Based on the "physical workshop-virtual workshop cloud platform" associated fusion technology, including the multisource multitype data collection, planning and packaging, and associated heterogeneous sensor layout optimization with data upload and cloud platform.

(3) Intelligent service technology based on digital twin data, including multiheterogeneous data fusion, multifactor collaborative production analysis, data prediction and maintenance strategy of workshop multisource disturbance, optimization of energy consumption, time, and other objectives in the whole life cycle scheduling process of products. It is precisely based on the functional guarantee provided by digital twin that the scheduling cloud platform can operate normally and achieve the unified matching of "demand-constraint-simulation-target-perturbation-adjustment." Otherwise, the cloud platform will lose its role and find it difficult to play the scheduling function. The process of the integration of digital twinning and shop scheduling is shown in Figure 2.

The scheduling mode of the cloud platform based on digital twin technology is different from the traditional scheduling mode and the traditional production scheduling mode. The scheduling mode of the cloud platform based on digital twin technology is an active scheduling mode, which actively selects or changes the production scheduling scheme according to different situations [26]. Regular orders are assigned to the respective production workshop to choose suitable processing equipment for production, but in the respective production workshop production process, the low level of production workshop is restrained by their high level of production workshop and management, and the deployment of manufacturing resources is limited. The production scheduling mode of enterprises is shown in Figure 2.

The optimization of parts manufacturing resources under the dispatching cloud environment is a circular and three-dimensional configuration, which makes orders break through geographical location limitations and administrative management limitations. The order task is no longer hierarchical decomposition control, but coordinated decomposition. For example, you can first process with the machining parts products in the factory workshop working procedure, and the second part product process can add in other factory worker process to produce high-quality resources reasonable use in a different factory, to formulate the optimal mode of production. In addition, as the intelligent scheduling of the dispatching cloud platform uses intelligent technologies such as the Internet of Things and cloud computing, the dispatching cloud platform can monitor the production schedule of products in real time, and the processing route is unified formulated by integrating highquality resources of the dispatching cloud platform, so that each workshop can know each other's processing tasks in advance and get ready for production. Therefore, compared 


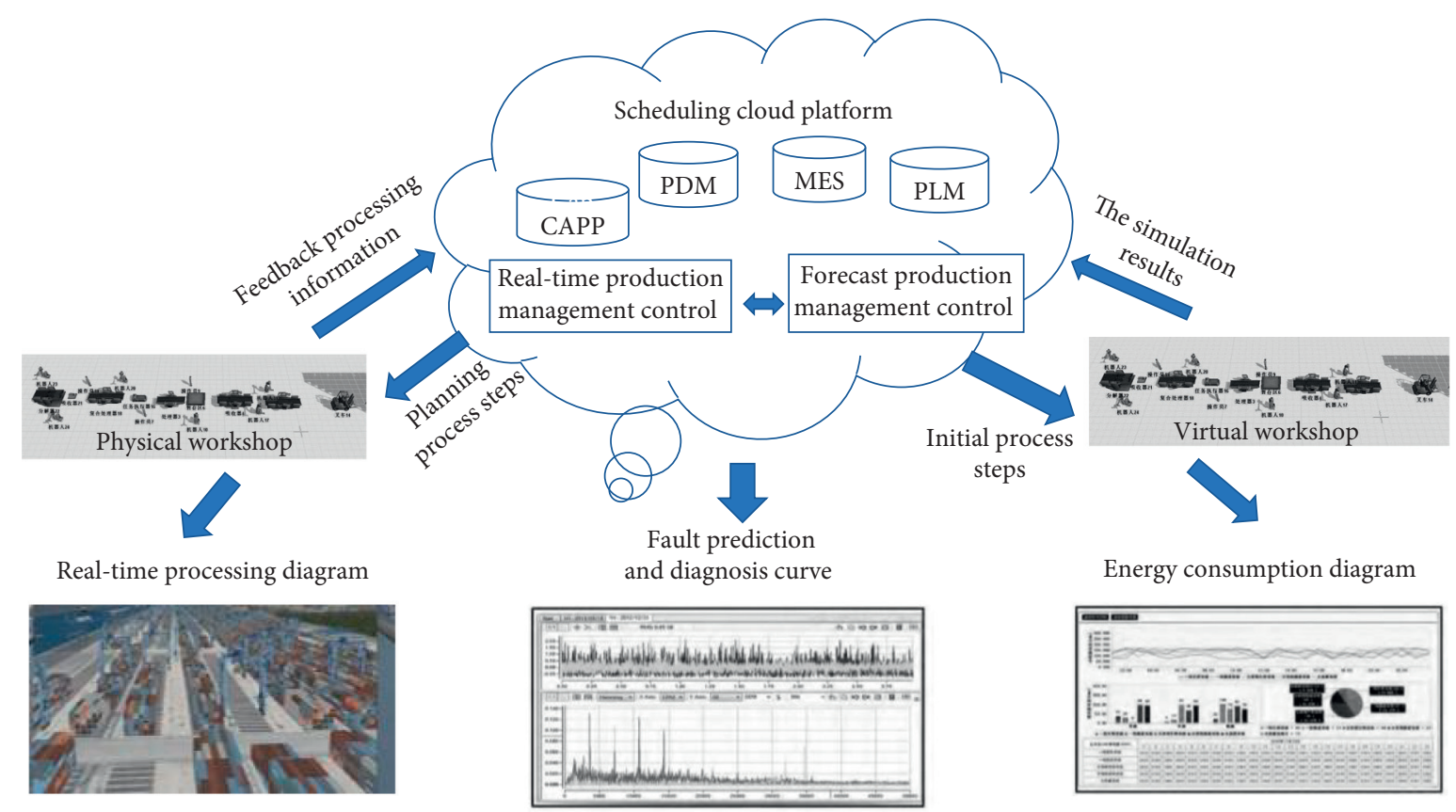

Figure 2: Fusion of digital twinning and shop scheduling.

with the production scheduling mode of traditional zeropart production enterprises, the production scheduling mode based on the scheduling cloud platform is more intelligent, can radiate to the whole life cycle, and is more suitable for the dynamic and changeable production environment.

\subsection{The Scheduling Platform Has Two Scheduling Process} Routes. The intervehicle tuning of the cloud platform is a complete process from the beginning of users' request for orders, through the decomposition of orders, to the generation of the initial scheduling scheme, to the adjustment of the initial scheme in the face of disturbances, and finally to the end of after-sales service after the delivery of orders [27]. All manufacturing resources such as equipment, software, status, and data of different factories will be uploaded to the scheduling cloud platform for storage, and technologies such as Computer-Aided Engineering Planning (CAPP), Product Data Management (PDM), Manufacturing Execution System (MES), and Product Lifecycle Management (PLM) will be integrated. There are two scheduling process routes in the model of the scheduling cloud platform after users' demands are raised:

(1) "Intelligent dispatching platform to physical virtual workshop" route: according to the specific needs of users, resources are matched in the production scheduling cloud platform repository, and a virtual scheduling model is established. Based on the virtual scheduling model, the simulation is put into production in the virtual workshop. The objective is to determine the effective algorithm model and determine the appropriate and efficient physical workshop production process sequence. At the same time, the physical workshop information is timely fed back to the scheduling cloud platform and the virtual workshop, which is accepted by the scheduling cloud platform. If the demand needs to be changed, the recycling from the scheduling cloud platform to the workshop will be performed; otherwise, the production task will be completed.

(2) "Intelligent scheduling cloud platform-virtual workshop, user-to entity shop," in particular, scheduling cloud without the required information in the database, finishes the first production part of the modeling and simulation and then feedback to the user, according to user requirements modeling to optimize production, until meeting user requirements, and then the workshop production orders, backups, and scheduling in the cloud, for the other similar orders.

\subsection{Intelligent Workshop Scheduling Cloud Platform Dynamic} Disturbance Strategy. In the production process of the parts in the workshop, due to the uncertainty of the production factors in the workshop, there will be emergency insertion, abnormal working conditions, and other uncontrollable disturbances. However, when the disturbance occurs, the formulation of the disturbance scheduling route will cause workshop shutdown and affect workshop efficiency [28]. Therefore, it is essential to predict the disturbance in the workshop.

Using big data analysis technology, the data stored in the life cycle monitoring system of scheduling cloud platform based on digital twin technology is analyzed to predict the disturbances that will occur, so as to develop a new production scheduling scheme in advance. Specifically, the scheduling 
cloud platform can calculate the impact of the initial operation according to the data analysis technology, determine whether there is a disturbance to adjust the current scheduling scheme, and establish the defined effect value. When the effect of the initial scheduling scheme with disturbance is worse than that of the definition, scheduling is required. However, as for the production disturbance of emergency insertion, it generally has a great influence on the initial scheduling scheme and will be directly rescheduled. The rescheduling module will first conduct a new scheme search on the scheduling cloud platform. If the corresponding part scheduling scheme can be found, it will be determined as a new scheduling scheme and then the new production scheduling scheme will be implemented. Otherwise, we simulate the new production scheduling plan in the virtual workshop, optimize the new production scheduling plan, make it meet the production requirements, and finally determine the production scheduling plan. The dynamic disturbance strategy diagram of part intelligent manufacturing workshop scheduling cloud platform is shown in Figure 3.

Step 1. The Production Planning Department shall conduct process design for personalized orders, generate cloud code of each process, process constraint parameters of the corresponding process, and upload equipment parameters and personalized order attribute parameters to the cloud. When an emergency order dynamic event occurs, the cloud scheduling platform first order depends on the type of history-related data for big data analysis evaluation to adjust the resource scheduling priority, the effectiveness of scheduling utility value, and evaluation to book value; if the effect is inferior to the preset value, continue to the original scheduling scheme to physical production workshop to continue production; if the effect is better than the preset effect, then enter the second step.

Step 2. Develop the rescheduling plan: The intelligent cloud scheduling platform shall make the preproduction plan according to the order delivery date, order priority, and manufacturing material inventory information and send the order to the twin workshop for simulation modeling to determine the new scheduling plan and implement the new production scheduling plan. In addition, simulate the new production scheduling plan in the virtual workshop, optimize the new production scheduling plan, make it meet the production requirements, and finally determine the production scheduling plan. The visual results of product modeling are displayed to users.

Step 3. Upload the rescheduled production scheduling plan to the cloud database, and timely feedback the scheduling arrangement to the real-time dynamic workshop for resource scheduling and resource coordination, so as to achieve on-time production and on-time delivery.

\section{Case Study}

4.1. Establish Scheduling Model. This case is a flexible manufacturing workshop for box parts of an enterprise. As shown in the figure, to construct the workshop framework, the first task is to establish the digital twin of FMC (fixedmobile convergence) automated production workshop on the virtual platform by referring to the production factors of the physical workshop and using digital modeling technology. Three aspects of model, constraint, and action should be taken into consideration when constructing digital twins.

In terms of model, the body parts of the FMC automation production workshop digital model of twin body are composed of workshop production factor model and correlation model. Workshop production factors include four parts and thus build the material library model and then the mechanical equipment model, environment model, and personnel model, but the mathematical models of the four still exist in isolation. To keep the connectivity between models, we also need to establish related models, such as the sensor model, the production scheduling model, and the product model. In terms of constraints, the constraints of digital twins in-the-box like zero-piece FMC automated production workshop consist of intermodel constraints, including equipment operation constraints, staff working time constraints, material supply constraints, and environmental constraints, which can ensure that the working mechanism of digital twins in the workshop can simulate and restore the physical workshop.

In terms of action, the action of a digital twin of the box part of FMC automated production workshop is composed of model action, which includes the working action of human, the operation action of equipment, the transport action of materials, and the adjustment action of environment. The most commonly used tools are Flexsim, Tecnomatix, Plant Simulation (the products of Siemens), etc. Combining the above three levels, the information of physical workshop and virtual workshop can be interactive and integrated. Finally, physical workshop and virtual workshop are connected to the factory scheduling cloud platform, which can allocate manufacturing resources, monitor the full life cycle of products, respond to multisource dynamic disturbances, and optimize the production process. After the model is established, its operation process is shown in the figure. The present industrial processing route is input into the virtual workshop for modeling processing in advance by the scheduling cloud platform. The assembly of the product is composed of $\mathrm{N}$ small processes, among which there is a certain sequential constraint relationship. Assembly line balance is how to allocate $\mathrm{N}$ processes to $M$ stations under the condition of satisfying production beats and constraints of sequence among processes, so as to achieve the highest balance rate of the production line. In Plant Simulation, a genetic algorithm can continuously optimize the feasible solution to find the optimal solution that meets the conditions. As the main goal of production efficiency, production line capacity is an important direction of production line planning and optimization for manufacturing enterprises. Since the production time is generally a distribution function rather than a certain value, and the failure rate of equipment is taken into account, the production capacity calculated by the common calculation method has a large deviation from the actual one. 


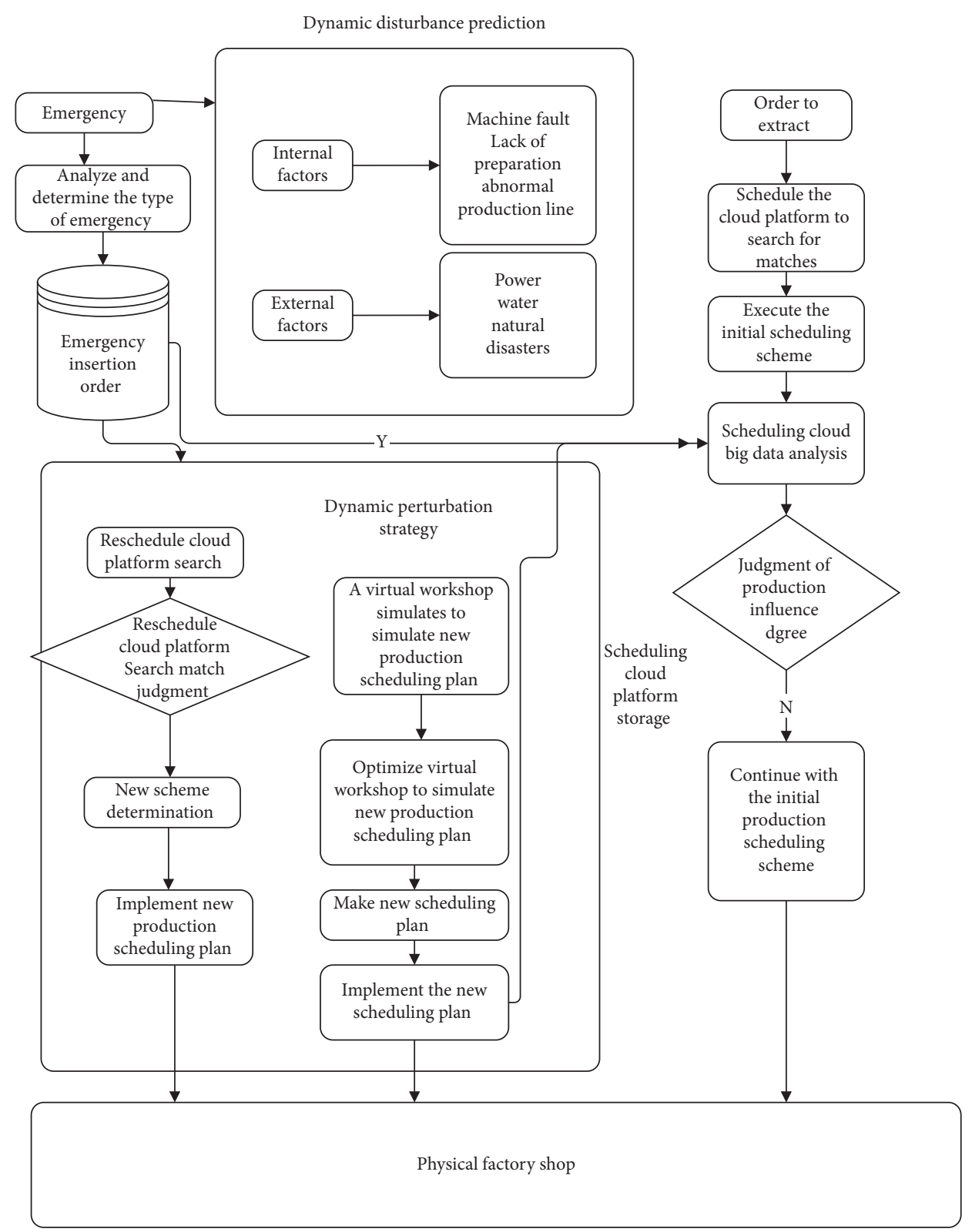

FIGURE 3: Intelligent manufacturing workshop scheduling cloud platform dynamic disturbance strategy diagram.

Plant Simulation can simulate the probability events such as distribution function and failure rate, so as to calculate the production capacity of the production line relatively accurately and find the bottleneck of the production line according to the simulation statistical results, thus pointing out the direction for the optimization of the production line.

4.2. Model Operation Stage. The optimal scheduling model based on digital twin technology is realized by Plant Simulation software. After the model is established, its operation process is shown in Figure 4. The present industrial processing route is input into the virtual workshop for modeling processing in advance by the scheduling cloud platform. After the processing has no problem, the scheduling cloud platform generates the working path of the manipulator and the working schedule of the manipulator. To output the work path and transport schedule to the physical workshop manipulator in accordance with the established path to carry out processing tasks, the work path and timetable can make the workpiece and equipment real-time connection and task matching, avoid the idling of processing equipment, and improve the work efficiency of the workshop. In the working process, the processing information and the scheduling cloud platform are integrated with each other. The scheduling cloud platform performs big data analysis, prediction, and judgment based on the feedback of historical processing data, predicts whether there are processing abnormalities, adjusts the production process parameters if there are any, and saves the processing data if there are none. 


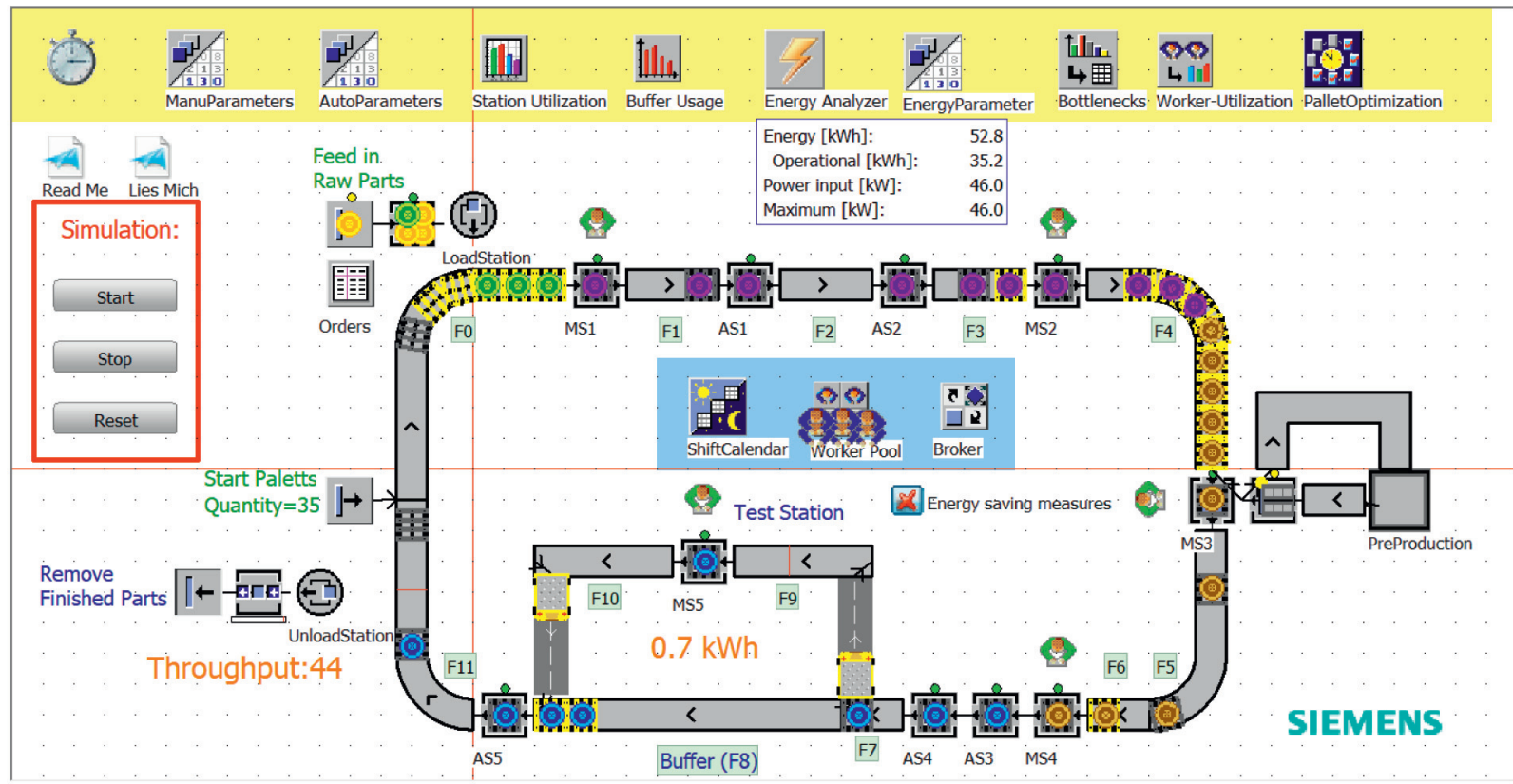

Figure 4: Model simulation diagram.

The optimal scheduling model based on digital twin technology is realized by Plant Simulation software, and random factors are added in the optimization process, so that the model can better simulate the real production situation. According to the running results in Figure 5, the working time ratio and machine utilization ratio of the scheduling policy system with digital twin technology have been greatly improved. The output of the product is 259 in the same simulation time, and the output rate is as high as 97\%. Meanwhile, the feedback data information of energy consumption can be monitored in real time, such as total energy consumption of $556.5 \mathrm{~kW} \cdot \mathrm{h}$, material handling energy consumption of $10.4 \mathrm{~kW} \cdot \mathrm{h}$, and maximum energy consumption of a single operating cycle of $41.9 \mathrm{~kW} \cdot \mathrm{h}$.

\subsection{The Advantage of Intelligent Scheduling Platform.} There are three main aspects as follows:

(1) Real-time parts processing process reflects the box parts FMC automation of production workshop products, the products from the design, production, and outbound, and in the process of the final product after all connectivity between the virtual workshop and physical workshop, the product information will be in the form of data and scheduling cloud platform interactive communion, to realize the product whole life cycle of monitoring.

(2) In terms of fault prediction and diagnosis, based on the reflection of the real-time machining process of parts, the dynamic disturbance in the production process is predicted and diagnosed, and the corresponding dynamic disturbance strategy is developed in advance. The production in the process of multisource dynamic disturbance is mainly divided into emergency single and machine fault; emergency insert sheet is due to the production task needs on the basis of the original order, additional order, scheduling cloud platform receiving orders, and additional order in advance; virtual workshop according to the additional order number and variety in the initial scheduling scheme on the basis of simulation optimization generates a new scheduling scheme. The realization of machine fault diagnosis is to embed sensors into production equipment in advance to detect various data, including location, weight, temperature, vibration, and flow rate. The big data analysis technology of tuning cloud platform is used to predict and diagnose faults, and the real-time data is used to determine which equipment needs to be repaired or changed. In this case study, the dynamic disturbance prediction diagnosis of the dispatch is mainly based on BP (Back Propagation) neural network. Under normal circumstances, the vibration operation signal of machinery and equipment in the workshop should show a relatively stable trend. However, the vibration signals collected by the dispatching cloud platform will deviate from the steady trend when the equipment is worn or fails unexpectedly. In case of wear or unexpected failure of the equipment, the vibration signal of the machine and equipment will deviate from the stable trend. At this time, according to the unstable signal received, the dispatch cloud platform will take treatment measures for the abnormal equipment in advance. Whether it is an emergency insertion or a machine failure, the scheduling cloud platform determines whether to execute the rescheduling module based on influence degree. If it does, the virtual workshop will make 

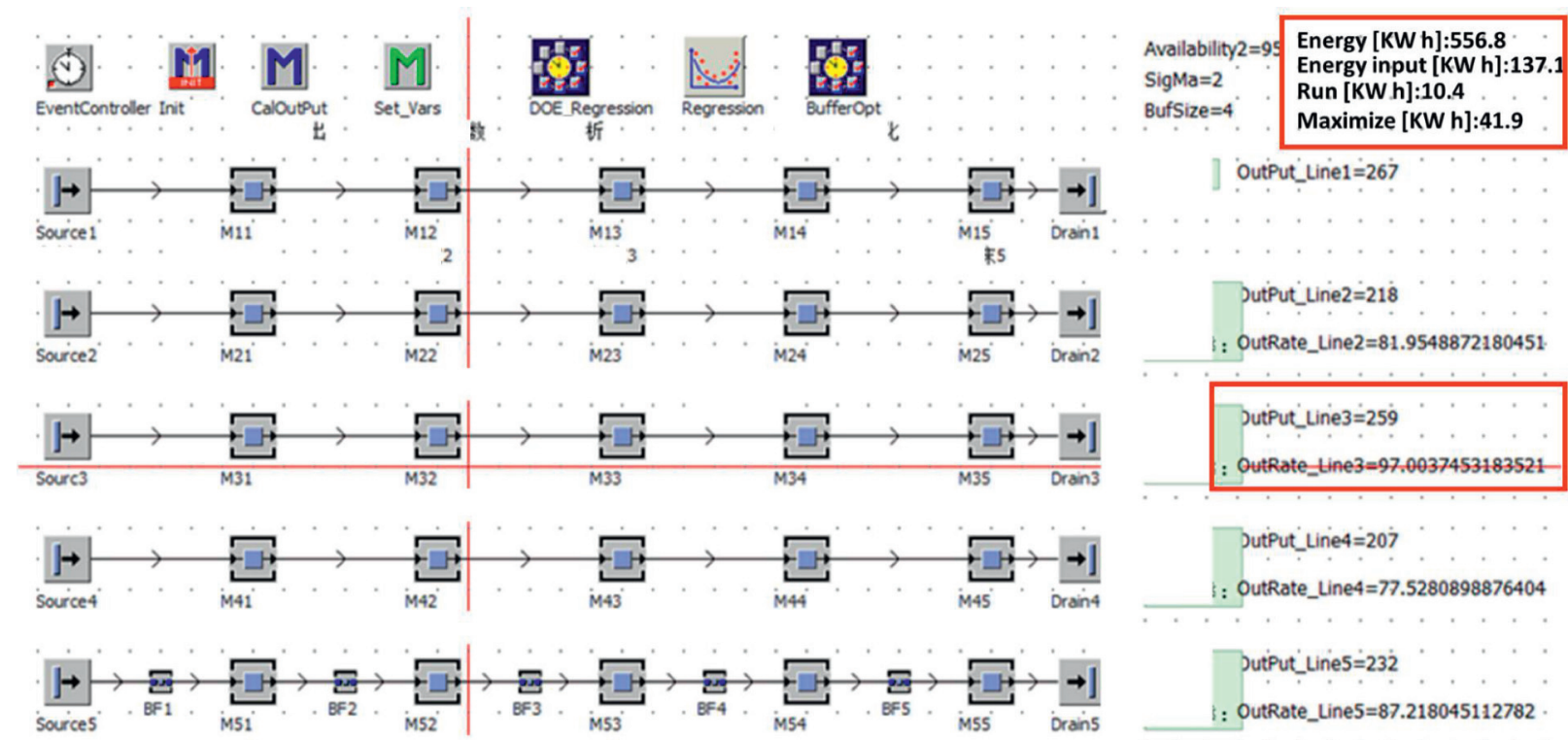

FIgURE 5: The result of the digital twin model.

process changes according to the disturbance situation. The virtual workshop will simulate production.

(3) The workshop scheduling is not to be ignored in the process of energy efficiency management; based on the technology of digital twin born real-time monitoring data, the data are multidimensional. and hierarchical cluster analysis reflects the workshop equipment energy consumption curve of each and clear understanding of the trends and characteristics of energy consumption, according to the trend and characteristics of shop scheduling process to make timely adjustment.

\section{Conclusion and Future Work}

Digital twins have great research potential in remote control, intelligent city management, health monitoring, and management. Although, in reality, the application of digital twin is still in its early stage, the industry is highly dependent on the sensor data, and there is a difficulty in global awareness of precise equipment, physical entity data is not enough detailed, a digital copy lacking limitation, algorithm, and processing architecture computing ability is limited, and many other issues, but we cannot deny that, in the coming wave of AIOT (AI + IoT) and intelligent manufacturing, the digital twin is an indispensable link; based on the "twin" physical entity + digital resources, optimization configuration system will become the ultimate digital development mode. We think that digital twin is still in its initial stage, and the future research will mainly focus on the following areas: (1) integration and optimization of the Internet of Things network among production factors in the physical workshop; (2) real-time interaction between the physical workshop and digital twin; (3) building and optimization of data analysis and prediction model and algorithm driven by big data; (4) development of more applications of digital twins and large data technology in manufacturing shop scheduling.

The future trends of cloud manufacturing services may cover the following research:

(1) The depth of the man-machine integration in the process of the supply of cloud services, including the process of cloud manufacturing service platform: "push" or "pull” process also includes user demand to realize the user-centric human-computer fusion deep degree and promote the formation of dynamic perception based on the personalized needs of research and development, manufacture, and the way of industrial organization. The cloud-oriented personalized knowledge service method considers user behavior, integrates personalized demand characteristics into cloud manufacturing service research, and provides research ideas for in-depth man-machine integration.

(2) Research on reliability and correctness of service portfolio: Since the reliability and correctness of service portfolio guarantee the efficiency and effect of demand task execution and are directly related to user perception and user satisfaction, it is necessary to carry out in-depth research on related technologies and algorithms.

(3) Construction of cloud manufacturing service autonomous management mode and knowledge reuse after the change of cloud manufacturing resource characteristics and attributes: service-oriented encapsulation needs to be carried out again. How to control the encapsulation process and improve the encapsulation efficiency through knowledge reuse is worthy of further study

(4) Comprehensive consideration of green manufacturing factors: In the context of national green production 
mode, more consideration of target factors such as high efficiency, low energy consumption, and recycling will be an important trend in cloud manufacturing service research.

\section{Data Availability}

No data were used to support this study.

\section{Conflicts of Interest}

The authors declare that they have no conflicts of interest.

\section{Acknowledgments}

This research work was supported by the Project of Research Planning Foundation on Humanities and Social Sciences of the Ministry of Education of China (No. 19YJA630105), the National Key Research and Development Project under Grant (No. 2020YFB1712802), and the National Key Research and Development Project under Grant (Nos. 2018YFB1700500 and 2018YFB1700503), and the Fundamental Research Funds for the Central Universities (No. N2106011).

\section{References}

[1] G. A. Yousef, B. Ali, X. Zhao et al., "A constraint multiobjective evolutionary optimization of a state-of-the-art dew point cooler using digital twins," Energy Conversion and Management, vol. 211, 2020.

[2] S. R. Yeratapally, P. E. Leser, J. D. Hochhalter, W. P. Leser, and T. J. Ruggles, "A digital twin feasibility study (part I): nondeterministic predictions of fatigue life in aluminum alloy 7075-T651 using a microstructure-based multi-scale model," Engineering Fracture Mechanics, vol. 228, Article ID 106888, 2020.

[3] R. Yeratapally Saikumar, E. Leser Patrick, D. Hochhalter Jacob, P. Leser William, and J. Ruggles Timothy, “A digital twin feasibility study (part I: non-deterministic predictions of fatigue life in aluminum alloy 7075-T651 using a microstructure-based multi-scale model," Engineering Fracture Mechanics, vol. 228, Article ID 106888, 2019.

[4] W. Luo, T. Hu, Y. Ye, C. Zhang, and Y. Wei, "A hybrid predictive maintenance approach for $\mathrm{CNC}$ machine tool driven by digital twin," Robotics and Computer-Integrated Manufacturing, vol. 65, Article ID 101974, 2020.

[5] Study data from University of Patras update understanding of applied sciences (a digital twin for automated root-cause search of production alarms based on KPIs aggregated from IoT), Science Letter, 2020.

[6] C. Zhang, W. Xu, J. Liu, Z. Liu, Z. Zhou, and D. T. Pham, “A reconfigurable modeling approach for digital twin-based manufacturing system," Procedia CIRP, vol. 83, 2019.

[7] E. Negri, L. Fumagalli, and M. Macchi, "A review of the roles of digital twin in CPS-based production systems," Procedia Manufacturing, vol. 11, pp. 939-948, 2017.

[8] F. Tao, F. Sui, A. Liu et al., "Digital twin-driven product design framework," International Journal of Production Research, vol. 57 , no. 12, 2019.

[9] H. Wang, H. Li, G. Luo, and C. Sun, "Digital twin based production design process and work load prediction method, computer integrated manufacturing systems," http://kns.cnki. net $/ \mathrm{kcms} /$ detail/11.5946.TP.20200506.1719.008.html.

[10] P. D. U. Coronado, R. Lynn, W. Louhichi, M. Parto, E. Wescoat, and T. Kurfess, "Part data integration in the shop floor digital twin: mobile and cloud technologies to enable a manufacturing execution system," Journal of Manufacturing Systems, vol. 48, pp. 25-33, 2018.

[11] M. A. Ali, R. Umer, and K. A. Khan, "A virtual permeability measurement framework for fiber reinforcements using micro CT generated digital twins," International Journal of Lightweight Materials and Manufacture, vol. 3, no. 3, pp. 204-216, 2020.

[12] B. Wang, G. Zhang, H. Wang, X. Jin, and K. Jiao, "Multiphysics-resolved digital twin of proton exchange membrane fuel cells with a data-driven surrogate model," Energy and AI, vol. 1, Article ID 100004, 2020.

[13] M. Zhang, T. Fei, and A. Y. C. Nee, "Digital twin enhanced dynamic job-shop scheduling," Journal of Manufacturing Systems, vol. 58, pp. 146-156, 2020.

[14] Q. Liu, J. Leng, D. Yan et al., "Digital twin-based designing of the configuration, motion, control, and optimization model of a flow-type smart manufacturing system," Journal of Manufacturing Systems, vol. 58, pp. 52-64, 2020.

[15] B. J. Ralph, A. Schwarz, and M. Stockinger, "An implementation approach for an academic learning factory for the metal forming industry with special focus on digital twins and finite element analysis," Procedia Manufacturing, vol. 45, pp. 253-258, 2020.

[16] B. R. Barricelli, E. Casiraghi, J. Gliozzo, A. Petrini, and S. Valtolina, "Human digital twin for fitness management," IEEE Access, vol. 8, pp. 26637-26664, 2020.

[17] L. He, W. Li, Yu Zhang, and Y. Cao, "A discrete multi-objective fireworks algorithm for flow shop scheduling with sequence-dependent setup times," Swarm and Evolutionary Computation, vol. 51, 2019.

[18] R. Alvarez-Valdes, A. Fuertes, J. M. Tamarit, G. Giménez, and R. Ramos, "A heuristic to schedule flexible job-shop in a glass factory," European Journal of Operational Research, vol. 165, no. 2, pp. 525-534, 2004.

[19] Y. Liu, H. Dong, N. Lohse, and S. Petrovic, "A multi-objective genetic algorithm for optimisation of energy consumption and shop floor production performance," International Journal of Production Economics, vol. 179, pp. 259-272, 2016.

[20] L. Yin, X. Li, L. Gao, C. Lu, and Z. Zhang, "A novel mathematical model and multi-objective method for the lowcarbon flexible job shop scheduling problem," Sustainable Computing: Informatics and Systems, vol. 13, 2017.

[21] H. Wang, Z. Jiang, Y. Wang, H. Zhang, and Y. Wang, "A twostage optimization method for energy-saving flexible job-shop scheduling based on energy dynamic characterization," Journal of Cleaner Production, vol. 188, pp. 575-588, 2018.

[22] G. Zhang, X. Shao, P. Li, and L. Gao, "An effective hybrid particle swarm optimization algorithm for multi-objective flexible job-shop scheduling problem," Computers \& Industrial Engineering, vol. 56, no. 4, pp. 1309-1318, 2008.

[23] Z. C. Li, B. Qian, R. Hu, L. L. Chang, and J. B. Yang, "An elitist nondominated sorting hybrid algorithm for multi-objective flexible job-shop scheduling problem with sequence-dependent setups," Knowledge-Based Systems, vol. 173, pp. 83-112, 2019.

[24] H. Mokhtari and A. Hasani, "An energy-efficient multi-objective optimization for flexible job-shop scheduling problem," Computers and Chemical Engineering, vol. 104, 2017. 
[25] Y. Wei, T. Hu, T. Zhou, Y. Ye, and W. Luo, "Consistency retention method for CNC machine tool digital twin model," Journal of Manufacturing Systems, vol. 58, pp. 313-322, 2020.

[26] W. Li, M. Rentemeister, B. Julia, D. Jöst, D. Schulte, and D. U. Sauer, "Digital twin for battery systems: cloud battery management system with online state-of-charge and state-ofhealth estimation," Journal of Energy Storage, vol. 30, Article ID 101557, 2020.

[27] B. Moya, I. Alfaro, D. Gonzalez, F. Chinesta, and E. Cueto, "Physically sound, self-learning digital twins for sloshing fluids," PLoS One, vol. 15, no. 6, Article ID e0234569, 2020.

[28] S.-J.Y. ChongWang, S.-i. Tanabe, and K. Ito, "Investigation of transient and heterogeneous micro-climate around a human body in an enclosed personalized work environment," Energy and Built Environment, vol. 1, no. 4, 2020. 